\title{
Can PET be performed without an attenuation scan?
}

\author{
Colin Jones, $\mathrm{BSc}^{\mathrm{a}}$ and Ran Klein, $\mathrm{PhD}^{\mathrm{b}}$ \\ a Department of Systems and Computer Engineering, Carleton University, Ottawa, ON, Canada \\ b Department of Nuclear Medicine, The Ottawa Hospital, Ottawa, ON, Canada
}

Received Aug 5, 2015; accepted Aug 5, 2015

doi:10.1007/s12350-015-0266-5

\section{See related article, pp. 1086-1097}

Positron emission tomography (PET) has emerged as the king of non-invasive molecular imaging, largely due to its ability to quantify tracer concentrations in units of $\mathrm{Bq} / \mathrm{mL}$. This ability is heavily reliant on accurate correction of coincidence photon attenuation. In cardiac imaging applications, including myocardial perfusion imaging (MPI), accurate and robust attenuation correction (AC) has been coveted as a means to distinguish between uptake deficits and attenuation artifacts. The notion that PET is able to deliver near perfect $\mathrm{AC}$, along with other advantages, has greatly contributed to the growth of cardiac PET in recent years.

From its early days, AC was an integral part of PET. Early instrumentation used weak, rotating transmission sources (e.g., ${ }^{68} \mathrm{Ge} /{ }^{68} \mathrm{Ga}$ or ${ }^{137} \mathrm{Cs}$ ) to measure attenuation along each line of response. The AC factors were then applied to the measured coincidence data to compensate for attenuation prior to image reconstruction. These transmission scans were lengthy and produced noisy data that propagated into the reconstructed image. With the introduction of hybrid PET-CT, x-ray computed tomography (CT) data could be acquired in a few seconds and manipulated to produce nearly noise-free AC estimates, ${ }^{1}$ and replaced traditional transmission scans as the de facto AC method in clinical PET. Recently, PET-MR hybrid systems have become available and rely on processing of magnetic resonance images (MR) to derive AC data. MR-based AC is a complex problem requiring further research and development, ${ }^{2}$ but has been

\footnotetext{
Reprint requests: Ran Klein, Department of Nuclear Medicine, The Ottawa Hospital, Ottawa, ON, Canada; rklein@toh.on.ca

J Nucl Cardiol 2016;23:1098-101.

$1071-3581 / \$ 34.00$

Copyright (c) 2015 American Society of Nuclear Cardiology.
}

demonstrated to produce equivalent clinical finding compared to PET-CT. ${ }^{3,4}$ While the nuclear imaging community welcomed these hybrid technologies due to the shorter scan times and the ability to co-register anatomical and functional information, it quickly became clear that certain challenges lay ahead with regards to AC.

Both CT and MR approaches suffer from misalignment problems due to patient motion between acquisition on attenuation and emission data. Rigidbody motion (e.g., of the head) can be effectively corrected using manual or automated registration methods. ${ }^{5}$ However, compensation for non-rigid patient motion is much more challenging and remains the topic of much research. In the context of nuclear cardiology, respiratory and cardiac motions further compound the challenge. Both CT and MR data are captured split second, resulting in mid-breath and mid-cardiac cycle images that do not fully correspond to PET data that is acquired over many breathing and cardiac cycles, even when emission data are gated (respiratory or cardiac). Breath-hold regiments during $\mathrm{CT}$ or MR data acquisition and cardiac triggering are common strategies to minimize motion artifacts within the attenuation image. Nevertheless cardiac imaging always requires verification and often correction to ensure optimal AC registration. Registration is performed with regards to the heart, often resulting in misregistration of other structures in the image (e.g., liver, lung, skeletal muscle and bone).

Respiratory or cardiac motion related AC misregistration can be partially accounted for using 4D-CT or -MR techniques; however, 4D techniques typically assume regular periodic motion such as uniform breath cycles and cardiac motion. ${ }^{6}$ Other, more practical limitations of these technologies have also been acknowledged. CT-AC can be a significant source of radiation to the patient compared to the tracer dose (e.g., $\sim 0.5 \mathrm{mSv}$ CT-AC dose vs $\sim 1 \mathrm{mSv}$ from a ${ }^{82} \mathrm{Rb} \operatorname{scan}^{7}$ ) especially if $4 \mathrm{D}-\mathrm{CT}$-AC is used to account for respiratory motion. ${ }^{16}$ PET-MR systems are currently prohibitively expensive, can increase the overall 
study length, and have significant counter indications (e.g., non-MR compatible implanted devices and claustrophobia).

\section{ESTIMATING ATTENUATION FROM THE EMISSION DATA}

Another approach to correct for attenuation, which was proposed over three decades ago, ${ }^{8}$ is to determine the attenuation information from the emission scan directly. Nuyts et al. explored this approach in 1999 using two algorithms: maximum likelihood (ML), and maximum a posteriori (MAP) iterative reconstructions using decreasing ordered subsets (OS). The results of both algorithms showed promise in comparison to no AC or standard AC, with MAP outperforming ML. ${ }^{9}$ Despite promising results, simultaneous estimation of attenuation and activity suffers from cross-talk, propagating errors between both images. ${ }^{10}$

Time of flight (TOF) information can be used to help localize updates during iterative reconstructions and reduce the cross-talk problem. With increasing prevalence of TOF capabilities in modern PET scanners, a resurgence in interest in extracting both attenuation and activity images from emission scan data has happened in the past few years. ${ }^{10}$ The trend in improved TOF resolution continues with enhancements to scintillation crystals, photomultipliers, and a move to new materials such as plastics that convert the gamma photons directly to voltages and also provide improved spatial resolution. ${ }^{11-15}$ As temporal and spatial resolutions within detectors increase, the ill-posed problem of reconstruction becomes less severe with positron range becoming the limiting factor.

With the improved TOF information of current scanners, the ML algorithm of Nuyts was reinvestigated by Rezaei et al. in 2012. ${ }^{10}$ The algorithm was extended to account for TOF, as maximum likelihood attenuation and activity (MLAA). While MLAA is able to reconstruct attenuation and activity images, the resulting images are subject to a scaling factor, which is due to estimating two related parameters - the estimated activity and the estimated attenuation. For a given measured sinogram, if the estimated activity is increased, then the estimated attenuation can also be increased to result in the same sinogram. Inaccurate scaling is unacceptable for absolute quantification with PET (e.g., standard uptake value interpretation, kinetic analysis, or gated imaging), but is of little concern for relative image interpretation (e.g., MPI or viability mismatch). Nevertheless this ambiguity can be resolved post reconstruction using attenuation and/or emission information from a known reference region (e.g., bed insert, surface marker, or tissue region) and applied as a scaling factor to produce true attenuation and activity values. ${ }^{10}$

\section{MLAA FOR CARDIAC PET}

In this installment of JNC, Presotto et al. investigate the use of MLAA on ${ }^{13} \mathrm{~N}$-ammonia MPI using a TOF scanner. Using a phantom experiment they demonstrate convergence of the MLAA algorithm which is slower than its non-MLAA counterpart. Nevertheless they conclude that convergence can be dramatically accelerated using a CT scan as an initial estimate of the attenuation map.

However, it would seem that the original misregistration issues that MLAA sought to address has reappeared, and therefore Presotto et al. go on to assess the impact of initial CT misregistration on image reconstruction. Averaged cine CT images with artificially induced misregistration as well as end-inspiration CT were used to evaluate whether global and local misalignment artifacts were eliminated using MLAA. While regular TOF reconstruction resulted in misregistration artifacts, MLAA reconstructed images, even after initial AC misregistration, were clinically equivalent to the reference images, which were reconstructed using accurately registered averaged cine CT-AC to account for respiratory and cardiac motions. Furthermore, endinspiration $\mathrm{CT}$ achieved similar results to averaged cine CT — a significant radiation dose reduction. This study provides good reason for excitement since PET without the need for separately acquired AC data offers exceptional promise.

\section{Static PET}

While low-dose CT-AC is typically performed at near end-diastole ( $\sim 70 \% \mathrm{R}-\mathrm{R}$ interval) using an mid- to end-expiration protocol, ${ }^{17}$ it may not accurately represent the average attenuation effects during PET acquisition. MLAA offers an image-specific cardiac and respiratory averaged AC.

\section{Gated PET}

Without the need for accurately registered AC data, gated (cardiac or respiratory) images can be reconstructed with phase-matched AC without the need to acquire 4D-CT, which currently delivers unacceptably high radiation dose ${ }^{16}$ and inaccurately assumes that respiratory motion during the $\mathrm{CT}$ is representative of breathing during PET.

\section{Dynamic PET}

In dynamic PET imaging (e.g., for myocardial blood flow quantification) MLAA may offer a unique solution to the patient motion and "cardiac creep" problems. These types of motion not only cause AC 
misregistration in part of the dynamic scan, but also erroneous region of interest sampling. Both artifacts lead to erroneous time-activity curves on which further analysis is dependent. MLAA may be able to correct for AC misregistration at each phase independently. Subsequent analysis of either the emission and/or attenuation image sequences could resolve the motion and then correct for it post reconstruction. Use of attenuation images may be advantageous over that of the activity images since attenuation is presumed constant, while the activity distribution changes between frames complicating motion detection.

\section{PET-MR}

MLAA may be a viable solution for generating reliable AC data from MR images, which do not directly correspond to photon attenuation values. MLAA may be able to refine the MR-derived attenuation maps (e.g., add dense bone structures) and complete missing image data in regions outside the MR field of view. ${ }^{17}$

Nevertheless, some caution with regards to MLAA is also warranted. In theory, MLAA can recover attenuation values only within the bounds of active contour ${ }^{18}$ as reflected by the phantom experiment described by Presotto et al. in which the phantom was wrapped in radioactive-soaked pads to emulate tracer uptake by skin. In the patient studies, Presotto et al. investigated the use of ${ }^{13} \mathrm{~N}$-ammonia, which distributes to tissues other than the heart (e.g., lungs and liver), but only marginal uptake is present in the skeletal muscles and skin. The authors did make an adjustment to the MLAA algorithm, wherein they added false counts to avoid the boundary condition, along with a prior favoring tissue or air attenuation values at the body contour. It is unclear how MLAA would perform with other tracers with different biodistribution, or in early time frames of dynamic imaging in which the activity is contained in the major blood vessels and has not yet perfused the organs.

Another point for contemplation is the effect of acquired counts and their relation to image noise and robustness of the MLAA algorithm. With MLAA roughly twice as much information must be resolved from the emission data: activity and attenuation. A tracer with fast decay (e.g. ${ }^{82} \mathrm{Rb}$ or ${ }^{15} \mathrm{O}$-water) and/or low retention may require higher injected activities to achieve equivalent image quality. A similar concern applies to short time frames $(<10 \mathrm{~s})$ in dynamic imaging.

As reported in earlier literature, an important drawback of MLAA is the scaling factor, which prevents quantitative analysis of the resulting images. This hinders MLAA from being used in dynamic (e.g., quantification of blood flow or metabolic rate) and gated studies, in which each image in the series must be on the same absolute scale. The authors propose two methods to resolve the inconsistent scaling factors: to use an external reference point source, or to use the entire data series to determine $\mathrm{AC}$, and then apply the $\mathrm{AC}$ to each frame. Nevertheless, to the best of our knowledge, no scaling method has been validated to date.

Before the work of Presotto et al. could be considered for clinical applications, larger studies would need to be performed. For example, in one experiment, only five patient studies with defects were examined, While three of the five resulted in similar images between the reference and MLAA, the other two showed minor differences. With such a small sample size, it cannot be determined if the results are indicative of the expected results, or simply due to chance. This limitation was acknowledged by the authors.

With these exciting advantages and points of concern in mind, cautious enthusiasm may be the most appropriate response to MLAA at this point in time.

\section{Disclosure}

R. Klein receives license revenues from the sale of FlowQuant. Consultant for Jubilant-DraxImage and receives royalties from rubidium generator technology licenses. C. Jones has nothing to disclose.

\section{References}

1. Townsend DW. Dual-modality imaging: Combining anatomy and function J Nucl Med 2008;49:938-55.

2. Yoo HJ, Lee JS, Lee JM. Integrated whole body MR/PET: Where are we? Korean J Radiol 2015;16:32-49.

3. Eiber M, Takei T, Souvatzoglou M, Mayerhoefer ME, Fürst S, Gaertner FC. Performance of whole-body integrated 18F-FDG PET/MR in comparison to PET/CT for evaluation of malignant bone lesions. J Nucl Med 2014;55:191-7.

4. Nensa F, Poeppel TD, Beiderwellen K, Schelhorn J, Mahabadi AA, Erbel R. Hybrid PET/MR imaging of the heart: Feasibility and initial results. Radiology 2013;268:366-73.

5. Alessio AM, Kinahan PE, Champley KM, Caldwell JH. Attenuation-emission alignment in cardiac PET/CT based on consistency conditions. Med Phys 2010;37:1191.

6. Reader AJ, Verhaeghe J. 4D image reconstruction for emission tomography. Phys Med Biol 2014;59:R371-418.

7. Hunter CRRN, Hill J, Ziadi MC, Beanlands RSB, deKemp RA. Biodistribution and radiation dosimetry of $(82) \mathrm{Rb}$ at rest and during peak pharmacological stress in patients referred for myocardial perfusion imaging. Eur $\mathbf{J}$ Nucl Med Mol Imaging 2015;42:1032-42.

8. Censor Y, Gustafson DE, Lent A, Tuy H. A new approach to the emission computerized tomography problem: Simultaneous calculation of attenuation and activity coefficients. IEEE Trans Nucl Sci 1979;26:2775-9.

9. Nuyts J, Dupont P, Stroobants S, Maes A, Mortelmans L, Suetens P. Evaluation of maximum-likelihood based attenuation correction in positron emission tomography. IEEE Trans Nucl Sci 1999;46:1136-41. 
10. Rezaei A, Defrise M, Bal G, Michel C, Conti M, Watson C. Simultaneous reconstruction of activity and attenuation in time-offlight PET. IEEE Trans Med Imaging 2012;31:2224-33.

11. Gola A, Ferri A, Tarolli A, Zorzi N, Piemonte C. SiPM optical crosstalk amplification due to scintillator crystal: Effects on timing performance. Phys Med Biol 2014;59:3615-35.

12. Nemallapudi MV, Gundacker S, Lecoq P, Auffray E, Ferri A, Gola A. Sub-100 ps coincidence time resolution for positron emission tomography with LSO: Ce codoped with Ca. Phys Med Biol 2015;60:4635-49.

13. Marino N, Baronti F, Fanucci L, Saponara S, Roncella R, Bisogni MG. A multichannel and compact time to digital converter for time of flight positron emission tomography. IEEE Trans Nucl Sci 2015;62:814-23.

14. Galetta G, Deleo R, Garibaldi F, Grodzicka M, Lagamba L, Loddo $\mathrm{F}$, et al.: Coincidence resolution time of two small scintillators coupled to high quantum-efficiency photomultipliers in a PET-like system. In: EPJ web of conferences, 2014, vol. 66, p. 10010.

15. Raczyński L, Moskal P, Kowalski P, Wiślicki W, Bednarski T, Białas $\mathrm{P}$, et al. Novel method for hit-position reconstruction using voltage signals in plastic scintillators and its application to positron emission tomography. Nucl Instrum Methods Phys Res A 2014;764:186-92.

16. Pan T, Mawlawi O, Nehmeh SA, Erdi YE, Luo D, Liu HH. Attenuation correction of PET images with respiration-averaged CT images in PET/CT. J Nucl Med 2005;46:1481-7.

17. Nuyts J, Bal G, Kehren F, Fenchel M, Michel C, Watson C. Completion of a truncated attenuation image from the attenuated PET emission data. IEEE Trans Med Imaging 2013;32:237-46.

18. Defrise M, Rezaei A, Nuyts J. Time-of-flight PET data determine the attenuation sinogram up to a constant. Phys Med Biol 2012;57:885 